\title{
A iniciação científica no ensino superior
}

\section{Scientific initiation program in high school}

\section{Marcos Jorge ${ }^{[a]}$, Tiago Santos Telles ${ }^{[b]}$, Ana Carolina Patrocino ${ }^{[c]}$}

[a] Doutor em Educação pela Universidade Estadual de Campinas (Unicamp), professor do Programa de Pós-Graduação em Educação da Universidade Estadual de Londrina (UEL), Londrina, PR - Brasil, e-mail: mjeduc@gmail.com

[b] Economista, Londrina, PR - Brasil, e-mail: tiagotelles@yahoo.com.br

[c] Pedagoga, Londrina, PR - Brasil.

\section{Resumo}

O objetivo deste artigo é apresentar um panorama da Iniciação Científica (IC) e do PIBIC nas universidades estaduais do Paraná, focando principalmente a Universidade Estadual de Londrina (UEL), entre 1995 e 2005. Para tanto, a partir dos Anais dos Encontros Anuais de Iniciação Científica (EAIC), é possível observar que a IC se institucionaliza de forma gradual no conjunto das instituições analisadas, e também é significativo o movimento crescente de comunicações apresentadas ao longo do período ao lado de uma relativa instabilidade no número dessas comunicações. Atualmente, as cinco universidades estaduais paranaenses 
estão fortemente envolvidas com projetos de IC, mas cabe destacar a primazia da Universidade Estadual de Maringá (UEM) e da UEL.

Palavras-chave: PIBIC. EAIC. Pesquisa. Ensino superior.

\section{Abstract}

The objective of this article is to present a survey of the Scientific Initiation (SI) and of the PIBIC in the states universities of Parana with a focus in Universidade Estadual de Londrina (UEL), between 1995 and 2005. In order to do that, from Annals of the Annual Meeting of Scientific Initiation (EAIC) is possible to observe that the SI is institutionalize of gradual form in the set of the analyzed institutions, and also is significant the increasing movement of communications presented to the long period to the side of a relative instability in the number of these communications. Currently, the five institutions are strong involved with projects of SI with a leadership to the Universidade Estadual de Maringá (UEM) and UEL.

Keywords: PIBIC. EAIC. Research. High education.

\section{Introdução}

Com o advento da chamada “sociedade do conhecimento", questões ligadas à educação superior ganharam relevância considerável enquanto objeto de investigação, temas e enfoques dos mais variados. Dentre estes, é possível citar a problematização de aspectos de caráter macroestruturais, tais como a democratização do acesso, a centralização, a descentralização, as formas de financiamentos, as características regionais, a organização e gerenciamento interno das instituições, e o grau de envolvimento com o entorno social.

O pressuposto contido na expressão "sociedade do conhecimento" universaliza a tese da emergência e consolidação de um modelo de organização econômica que engendra na produção, armazenamento e controle da disseminação da informação, principalmente aquela de base 
técnico-científica, com considerável potencial para o investimento que visa lucros, o seu principal substrato, a sua base de existência.

O aceite dessa proposição valida também a alegação de que tal paradigma somente é possível de se realizar a partir do consentimento dos indivíduos e da sociedade, da necessidade de mudanças ou transformações (acompanhadas de atributos como radicais, urgentes e inevitáveis) que se expressariam no abandono ou negação de "antigos" valores e a incorporação de outros tantos mais coetâneos ao mundo informacional, à construção e institucionalização das futuras gerações de novas expectativas de futuro. Dentre estas, destaca-se a do convencimento sobre as incertezas do mundo do trabalho, com empregos cada vez mais instáveis e fluidos, com a onipresente busca por formação educacional ao longo de toda a vida, ou seja, a aceitação de que o conhecimento adquirido tem duração limitada, um ciclo de vida compatível com a instantaneidade, e que Baumann (2002, p. 51) chamou de "mercado do conhecimento", marcado pela comercialização da novidade, do inédito, do efêmero e que já não mais se referencia pelos "hábitos arraigados, as estruturas cognitivas sólidas, e a preferência por valores estáveis".

O capitalismo doravante dependente do conhecimento ou o conhecimento transmutando-se em força produtiva é algo que, segundo Giannotti (2002, p. 107), "vem conformar o mercado de forma inédita na história moderna".

O debate em torno da reestruturação dos sistemas universitários não é um fenômeno isolado, está presente no interior das sociedades dos países de economia mais dinâmica de todos os continentes. Dada a abrangência e o alcance do tema, talvez fosse possível caracterizá-lo como um assunto globalizado, embora as análises sobre seu desempenho, sucessos, deficiências e propostas de intervenção variem de um país para outro. Na América Latina, Catani e Oliveira (2000, p. 29) observam que os diagnósticos e as proposições para mudanças se pautam em "documentos internacionais orientadores dos modelos e das reformas de educação superior".

Tais documentos se constituem de estudos ou análises feitas por agências multilaterais, como o Banco Mundial, a Unesco e a referência europeia, expressa no Relatório Attali. Para Catani e Oliveira (2000, p. 45), o encaminhamento dos debates a partir dessas fontes evidenciam que "as temáticas, as críticas, as tendências e as políticas e estratégias mais significativas na América Latina, revelam o alto grau de subordinação dos países em 
relação às orientações dos organismos internacionais, particularmente do Banco Mundial".

Dessa forma, o sistema de ensino superior, abrangendo os setores público e privado, interage com a sociedade e absorve integral ou parcialmente os projetos sociais em curso e, operando dentro dos limites impostos, procura responder às demandas exigidas por essa mesma sociedade. Além disso, os paradigmas que balizaram o chamado "setor terciário da educação" sofrem pressão para se readaptarem a essa nova "fase" do capitalismo ou "sociedade do conhecimento".

Embora tema importante, a discussão sobre o papel exercido por tais instituições no encaminhamento da política educacional latinoamericana para o ensino superior extrapola os objetivos deste trabalho e deve ser discorrido em outra oportunidade.

Nossa intenção é destacar as políticas em torno da melhoria da qualidade do ensino de graduação, entendendo que tais políticas, particularmente o Programa Institucional de Bolsas de Iniciação Científica (PIBIC) (1999), estão inseridas no contexto mais amplo, no sentido de introduzir um dinamismo estrutural no sistema de ensino superior para que este possa se relacionar e operar mais próximo às demandas da sociedade civil, seja no sentido de produzir efeitos na economia, com o desenvolvimento de programas de ciência e tecnologia, na formação de recursos humanos em diferentes níveis de especialização, mas também no encaminhamento de propostas que promovam a democracia, a cidadania e a equidade social. (SANTOS, 2003).

\section{A iniciação científica e o PIBIC}

Em fins dos anos 80, o Estado brasileiro tomou a iniciativa de expandir para o conjunto do sistema universitário (no nível da graduação) a experiência do PIBIC, que existia desde a década de 50, quando da institucionalização do Conselho Nacional de Desenvolvimento Científico e Tecnológico (CNPq).

Duas metas se destacam no encaminhamento do PIBIC: primeiro, a formação precoce nos alunos de graduação de uma mentalidade científica por meio de projetos de Iniciação Científica (IC, 2000) e, segundo, uma inserção mais rápida dos alunos participantes de IC no mestrado ou doutorado, onde se espera uma redução no seu tempo de titulação. 
O destaque e relativo sucesso do PIBIC ao longo do tempo geraram uma percepção de que seu modelo poderia trazer melhorias ao ensino básico e, atualmente, uma versão do programa Iniciação Científica Júnior está sendo implementada no ensino médio de alguns Estados, entre eles, o Paraná.

Contraditoriamente, tal iniciativa que virtualmente congrega muitas implicações positivas em termos sociais e econômicos para o país e a sociedade local, parece não mobilizar integralmente a academia e os pedagogos, em particular, como instrumento de apoio profissional e objeto de investigações sistematizadas. Via de regra, a literatura sobre o tema registra esparsas reflexões críticas de acadêmicos ou balanços avaliativos originados de órgãos oficiais de fomento à pesquisa (BRIDI, 2004).

É na região Sudeste, na qual se concentra o polo universitário e de produção de ciência e tecnologia mais destacado do país, que o PIBIC tem longa tradição, e é de suas universidades que podemos encontrar a maior parte dos trabalhos que procuram avaliar seu gerenciamento e resultados. Paulatinamente, estudos de outras regiões começam a despontar, revelando concordâncias em alguns aspectos e também particularidades regionais. (YAMAMOTO; FERNANDES JUNIOR, 2002).

$\mathrm{Na}$ avaliação de Fava-de-Moraes e Fava (2000, p. 75), o PIBIC traz mais ganhos do que riscos para a instituição e para o aluno bolsista. Enumeram, ainda, algumas destrezas intelectuais e práticas cotidianas que este adquire ao longo da IC, como "a fuga da rotina e da estrutura curricular", o manejo competente dos sistemas de referências e bases de dados eletrônicas, desenvolve "capacidades diferenciadas nas expressões oral e escrita e nas habilidades manuais" e um dos aspectos mais positivos é o contato com um professor-orientador, geralmente aquele que ministra a disciplina cujo aluno "tem mais simpatia e paladar". Os autores apontam que vivenciar a IC leva o bolsista a "perder o medo, não ter pânico do novo", ou seja, estimula o espírito crítico e independente, além da autonomia e autoconfiança, condições essenciais para o fazer científico.

O estudo de Velloso e Velho (2001, p. 12) sugere que o PIBIC realmente propicia condições favoráveis para a inserção do aluno bolsista na pós-graduação. Observaram que " $22 \%$ dos mestrandos que não tiveram bolsa de graduação também nunca tiveram de mestrado" e encontraram forte correlação entre alunos bolsistas de IC e o seu "ingresso mais rápido no mestrado"; apontam que os "ex-ICs" demoram em média 2,4 anos após 
a graduação para chegar ao mestrado enquanto que entre os não bolsistas esse tempo é de 6,8 anos. Quanto à produção acadêmica discente, os autores observaram que " $63 \%$ dos mestrandos que tiveram bolsa de IC publicaram um ou mais resumos antes de ingressar no curso [mestrado] que freqüentavam", ao passo que apenas $45 \%$ dos que nunca participaram da IC o fizeram.

Observamos também uma análise positiva do PIBIC no trabalho de Silva e Cabrero (1998, p. 63), que apontam um aumento constante nas verbas do Programa ao longo da década de 90, que chega a alocar quase um terço das "despesas realizadas no país, com formação de recursos humanos e pesquisa". As bolsas de IC correspondiam a 27,04\%, sendo suplantadas pelo Mestrado (34,29\%) e Doutorado (33,13\%).

Com a autonomia propiciada pela Lei de Diretrizes e Bases (LDB), a partir de 1996 apareceram formas alternativas no gerenciamento e operacionalização do sistema de IC. Muitas universidades a implantaram nos moldes do PIBIC e foram registradas experiências de diversificação no seu encaminhamento, como ocorre no curso de Medicina da Universidade Federal Fluminense, no qual o aluno faz pesquisa e ganha créditos ao invés da bolsa (CARDOSO, 2004).

A premissa de que a IC tem desdobramentos na melhoria da qualidade do ensino de graduação é corroborada por Castro (20022007). O pesquisador registra esforços em ampliar conteúdos de história da ciência e metodologia científica em diversos cursos desse nível de ensino. Relatos de Maldonado e Paiva (2002) asseveram sobre o papel da IC na formação do nutricionista em quatros universidades públicas do Rio de Janeiro ${ }^{1}$.

Porém, enquanto trabalho coletivo que envolve variáveis complexas, como as diferenças regionais, a cultura da pesquisa, as limitações de verbas, o PIBIC enfrenta problemas. As pesquisas de Breglia (2001) e Bridi (2004) registraram as críticas dos alunos que vivenciaram a IC.

Carvalho e Angelini (1996) e Setton (2003) sugerem evidências de que o PIBIC deve periodicamente passar por avaliação interna da instituição. Os autores apontam que a IC tem relativa influência no desempenho discente (na graduação), com consequências negativas, tanto para instituição como

${ }^{1}$ Universidade do Estado do Rio de Janeiro (UERJ), Universidade Federal do Rio de Janeiro (UFRJ), Universidade Federal Fluminense (UFF) e Universidade do Rio de Janeiro (UNI-RIO).

Rev. Diálogo Educ., Curitiba, v. 10, n. 30, p. 441-457, maio/ago. 2010 
para o aluno, como o abandono de projetos e evasão do curso, motivados por frustrações pessoais e de expectativas profissionais. Tais avaliações são corroboradas pelos trabalhos de Souza, Oliveira e Gonçalves (2001) e Villas Bôas (2003).

Desta forma, se faz necessário estimular internamente, em todo o sistema educacional, iniciativas capazes de compatibilizar desempenho eficiente e retorno social. Para isso, é preciso intensificar a busca por instrumentos de avaliação, qualitativos e quantitativos, bem como ações corretivas das políticas públicas para o ensino superior, inclusive o PIBIC (AMARAL, 2004; VIANNA, 2004).

Sobre os aspectos das positividades auferidas ao PIBIC, Yamamoto e Fernandes Junior (2002) apresentam um balanço do primeiro quinquênio do Programa na Universidade Federal do Rio Grande do Norte (UFRN) desde sua implantação, em 1992, constatando que os resultados foram "extremamente positivos".

Os autores apontam que o número de professores envolvidos em atividades de pesquisas cresceu e "aumentou também a produção científica, ao menos em termos de divulgação interna”. Observam que na avaliação dos professores os pontos fortes a serem estimulados são "as habilidades mentais de comparação, julgamento, criação e investigação de problemas" que têm íntima relação no envolvimento com a pesquisa sistematizada, além de instigar a "criatividade, a curiosidade e a atitude crítica dos alunos". Os autores observam, a exemplo de Fava-de-Moraes e Fava (2000, p. 75), que a IC contribui para "uma atitude de independência e autonomia diante do conhecimento e das questões do cotidiano".

\section{Características do sistema estadual de ensino superior do Estado do Paraná}

O sistema de educação superior do Estado do Paraná ${ }^{2}$ reúne 151 instituições de ensino superior (IES), 22 públicas e 129 privadas, o que representa $50 \%$ do conjunto da região Sul ${ }^{3}$. Vale observar que para o conjunto

\footnotetext{
${ }^{2}$ O Estado do Paraná, capital Curitiba, ocupa uma área de 199.500 km2 (2,3\% do território brasileiro) e conta com uma população estimada em 9.564 milhões de habitantes $(5,45 \%$ da população brasileira).
} 
das instituições públicas (federal, estadual e municipal) da região, $61,11 \%$ são paranaenses e, dentre o universo das instituições públicas estaduais, verifica-se que 94,44\% estão em território paranaense (OECD, 2006).

Para os padrões de um país em desenvolvimentoe proporcionalmente à suas dimensões geográficas, contingente populacional, participação no PIB, o sistema estadual paranaense de ensino superior é, no mínimo, portentoso. Conforme mostra a Tabela 1, o Estado do Paraná possui seis universidades (cinco das quais possuem outros câmpus além da sede), sete faculdades e reúne ainda três hospitais universitários.

Tabela 1 - Número de cursos de graduação nas IEES paranaenses (2005)

\section{INSTITUIÇÕES ESTADUAIS DE ENSINO SUPERIOR (IEES)}

CURSOS

Universidade Estadual de Londrina (UEL)

Universidade Estadual de Maringá (UEM)

Universidade Estadual do Oeste do Paraná (Unioeste)

Universidade Estadual do Centro-Oeste (Unicentro)

Universidade Estadual de Ponta Grossa (UEPG)

Universidade Estadual (UENP)

Faculdade Estadual de Educação Ciências e Letras de Paranavaí (Fafipa)

Faculdade Estadual de Filosofia Ciências e Letras de Paranaguá (Fafipar)

Faculdade Estadual de Ciências e Letras de Campo Mourão (Facilcam)

Faculdade Estadual de Ciências Econômicas de Apucarana (Fecea)

Faculdade Estadual de Filosofia Ciências e Letras de União da Vitória (FAFI-UV)

Faculdade de Artes do Paraná (FAP)

Fonte: Elaborado pelo autor, a partir dos dados da SETI/PR, 2005.

\footnotetext{
${ }^{3}$ A região Sul brasileira tem de $576.980 \mathrm{~km} 2$ (6,8\% do território nacional), sendo formada pelos Estados do Paraná, Santa Catarina e Rio Grande do Sul. Sua população, de 25.108 milhões de habitantes, está concentrada na zona urbana $(80,93 \%)$ e corresponde a $14,95 \%$ da população do país.
} 
O sistema oferece 265 cursos de graduação (na área de educação somam 27 cursos, sendo 24 de Pedagogia, 1 de Normal Superior, e 2 de formação de professores para atuação específica, 1 de arte-educação e 1 de educação para o campo), 66 cursos de Mestrado, 17 de Doutorado e 315 cursos de Especialização, que somam 85.400 alunos. O corpo de funcionários integra 5.189 docentes e 8.458 técnicos administrativos (SETI, 2007).

Em relação ao país, o sistema de ensino superior da região Sul e do Paraná, em particular, consolidou-se tendo como característica seu forte enraizamento no interior dos Estados.

Os dados da Tabela 2 mostram que essa interiorização é aproximadamente de um terço para o país e dois terços para a região Sul, ou seja, mais de 85\% dos seus cursos de graduação desenvolvem-se no interior.

Tabela 2 - Número de cursos de graduação por categoria administrativa e localidade das IES no Brasil (2005)

\begin{tabular}{llllll}
\hline Localidade & $\begin{array}{l}\text { Sistema } \\
\text { público* }\end{array}$ & $\begin{array}{l}\text { Sistema } \\
\text { privado** }\end{array}$ & Total & Capital & Interior \\
\hline Brasil & 5460 & 5015 & 10475 & 3218 & 7257 \\
Região Sul & 818 & 1468 & 2286 & 333 & 1953 \\
Paraná & 325 & 259 & 584 & 150 & 434 \\
Santa Catarina & 262 & 441 & 703 & 73 & 630 \\
Rio Grande do Sul & 231 & 768 & 999 & 110 & 889 \\
\hline
\end{tabular}

Fonte: Elaborado pelo autor, a partir dos dados do MEC/INEP, 2005.

Notas: * Instituições federal e estadual.

** Instituições confessionais, comunitárias e filantrópicas.

O Estado do Paraná concentra significativa maioria de ofertas desses cursos em instituições públicas. Dos 325 cursos oferecidos pelo sistema público (federal e estadual), 265 são oferecidos por instituições estaduais (todos os câmpus estaduais são interiorizados) e representam $85,54 \%$ da oferta de cursos. As instituições federais respondem por $18,46 \%$ dos cursos, que somam 60 (58 na capital e 2 no interior).

No contexto paranaense, a oferta de cursos de graduação segue uma relação de equilíbrio entre os setores público e privado, dados que apresentam forte discrepância nos Estados de Santa Catarina e Rio Grande do Sul. 
Como já foi observado, diferentemente de outros Estados, como Rio de Janeiro, Minas Gerais e Rio Grande do Sul, nos quais se nota forte presença de universidades federais, o Estado do Paraná assume quase que a totalidade das responsabilidades com o sistema de ensino superior.

O Censo da Educação Superior (2005) revela que o sistema privado é o setor que registra a maior quantidade de vagas ofertadas. Esse fenômeno foi analisado localmente por Loch e Reis (2004), os quais, derivado desse processo, registraram que o sistema público absorveu uma minoria que corresponde a 107.078 matrículas, enquanto que na rede privada atingiu a marca de 205.020 matrículas (INEP, 2006). Nesse sentido, o Estado segue a tendência histórica nacional do atendimento de grande parte da demanda do nível superior ser preenchida pela rede privada ${ }^{4}$.

Juntos, os setores público e privado oferecem um percentual de matrículas que supera a representação populacional regional, conforme dados da Tabela 3.

Tabela 3 - Número de cursos de graduação por categoria administrativa e localidade das IES no Brasil (2005)

\begin{tabular}{lll}
\hline Região & \% Matrícula ensino superior & \% População nacional \\
\hline Sudeste & 49 & 42 \\
Sul & 19 & 15 \\
Centro-oeste & 10 & 07 \\
Nordeste & 16 & 28 \\
Norte & 06 & 08 \\
Total & 100 & 100 \\
\hline
\end{tabular}

Fonte: MEC/INEP - elaborada pelo autor.

\section{A iniciação científica na Universidade Estadual de Londrina}

As duas maiores universidades estaduais do Paraná, a Universidade Estadual de Londrina (UEL) e a Universidade Estadual de Maringá (UEM), localizam-se no norte do Estado e são dois importantes polos de dinamização regional.

\footnotetext{
${ }^{4}$ Para uma análise mais detalhada sobre a dinâmica do setor privado, ver: SAMPAIO, 2000.
} 
Como grande complexo universitário público, as duas instituições respondem conjuntamente por mais de um terço dos cursos de graduação do sistema estadual, aproximadamente $80 \%$ dos cursos de Mestrado e $100 \%$ do Doutorado, e reúnem corpo docente com maior titulação acadêmica, 69,1\% são doutores e 46,5\% são mestres (OECD, 2006). A população discente das duas instituições somadas totalizam 24.024 alunos de graduação e 6.296 na pós-graduação (stricto sensu e lato sensu) (Pró-Reitoria de Planejamento PROPLAN, 2007).

$\mathrm{O}$ engajamento das cinco universidades estaduais paranaenses com o PIBIC ocorreu ao longo da década de 90. A UEL, a UEM e a Universidade Estadual de Ponta Grossa (UEPG) aderiram ao Programa em 1990, entretanto, a UEM foi pioneira na implantação de uma Iniciação Científica institucional já em 1982, com o Programa de Bolsa de Pesquisa PPG/UEM, que gradualmente foi se incorporando ao PIBIC.

A partir de 1991, as três universidades trabalharam articuladas na execução da seleção dos projetos e dos orientadores e bolsistas, bem como a avaliação do PIBIC. Desse processo resulta anualmente o evento denominado Encontro Anual de Iniciação Científica (EAIC, 1998), no qual são apresentados à sociedade os resultados parciais ou finais dos projetos de pesquisa via IC. Inicialmente centrado nas IEES paranaenses, o EAIC, paulatinamente, vem atraindo a comunidade de IC de outros Estados do Sul e de outras regiões do Brasil.

Para este trabalho, tomamos como uma das fontes os Anais dos EAIC, entre os anos 1995 e 2005, podendo-se inferir que cada comunicação apresentada no evento representa pelo menos um projeto de IC, em sua grande maioria, financiado pelo PIBIC. No entanto, em relação à UEL, muitos projetos de pesquisa envolveram dois ou mais subprojetos de IC, dessa forma, acreditamos que os Anais dos EAIC são uma importante fonte de estudos.

A Tabela 4 mostra a evolução da apresentação de comunicações científicas provenientes de projetos de IC nos EAIC, na qual é possível observar dois fenômenos: primeiro, os números refletem certa instabilidade na participação das universidades na apresentação dos seus trabalhos de IC, por outro lado, nota-se um movimento ascendente no envolvimento de três (UEL, UEM e UEPG) das cinco universidades paranaenses, com a nítida proeminência da UEM. 
Tabela 4 - Trabalhos apresentados nos EAIC por instituição/ano

\begin{tabular}{|c|c|c|c|c|c|c|c|c|c|c|c|}
\hline \multirow{2}{*}{ IES } & \multicolumn{11}{|c|}{ Ano } \\
\hline & 1995 & 1996 & 1997 & 1998 & 1999 & 2000 & 2001 & 2002 & 2003 & 2004 & 2005 \\
\hline UEL & 173 & 222 & 196 & 207 & 209 & 309 & 444 & 570 & 516 & 609 & 485 \\
\hline UEM & 264 & 303 & 267 & 376 & 402 & 482 & 533 & 704 & 527 & 478 & 494 \\
\hline UEPG & 49 & 46 & 54 & 78 & 109 & 141 & 373 & 237 & 326 & 262 & 248 \\
\hline UNIOESTE & 01 & 17 & 15 & 36 & 161 & 129 & 170 & 271 & 323 & 390 & 279 \\
\hline UNICENTRO & 00 & 00 & 00 & 00 & 00 & 00 & 00 & 03 & 00 & 27 & 121 \\
\hline OUTRAS & 77 & 82 & 50 & 82 & 78 & 231 & 85 & 207 & 95 & 114 & 34 \\
\hline Total & 564 & 670 & 582 & 779 & 959 & 1292 & 1605 & 1992 & 1787 & 1880 & 1661 \\
\hline
\end{tabular}

Fonte: Elaborado pelo autor, a partir dos Anais do EAIC.

O ano de 1998 marca um momento importante, pois há um considerável incremento nessa participação com a inclusão da Unioeste e, posteriormente, da Unicentro. Movimento explicado em parte pela institucionalização da Fundação de Amparo à Pesquisa do Paraná (Fundação Araucária), que teveimpacto considerável em mobilizar os setores acadêmicos para a pesquisa. Tal fenômeno pode estar associado à corrente tese de que no Brasil a maior parte da pesquisa científica tem forte vínculo com o financiamento público e um de seus locus mais significativos é a universidade pública.

Embora significativos, os esforços e resultados do engajamento do sistema universitário público paranaense em alavancar a IC como um recurso didático-científico, os indicadores apresentados mostram quão distante o conjunto das cinco universidades está de algumas instituições universitárias brasileiras. Um exemplo é a Universidade Federal do Paraná (UFPR), que em seu Evento de Iniciação Científica (2005), expôs 929 comunicações, já a Universidade Estadual de Campinas (UNICAMP), no Congresso Interno de Iniciação Científica (2006), apresentou 1.293 trabalhos.

Ao buscarmos as áreas mais envolvidas com o PIBIC e, portanto, com projetos de IC, os dados da Tabela 5 apresentam destaque extremamente vantajoso para as Ciências Agrárias. Ao todo, o sistema público de ensino superior paranaense soma 18 cursos naárea: 7 de Agronomia, 5 de Zootecnia, 4 deMedicina Veterinária e 2 de Engenharia Agrícola. Vale observar que o Paraná é um dos Estados brasileiros onde o agronegócio é uma das atividades econômicas mais importantes, sendo possível conjecturar estar ocorrendo, com relativo sucesso, uma aproximação entre determinado setor produtivo e a academia. 
Tabela 5 - Trabalhos apresentados nos EAIC, por grandes áreas do conhecimento/ano

\begin{tabular}{|c|c|c|c|c|c|c|c|c|c|c|c|}
\hline \multirow{2}{*}{ Grandes áreas } & \multicolumn{11}{|c|}{ Ano } \\
\hline & 1995 & 1996 & 1997 & 1998 & 1999 & 2000 & 2001 & 2002 & 2003 & 2004 & 2005 \\
\hline $\begin{array}{l}\text { Ciências Exatas } \\
\text { e da Terra }\end{array}$ & 107 & 99 & 85 & 107 & 171 & 223 & 295 & 380 & 260 & 258 & 274 \\
\hline $\begin{array}{l}\text { Ciências } \\
\text { Biológicas }\end{array}$ & 135 & 127 & 126 & 164 & 173 & 182 & 262 & 279 & 233 & 327 & 308 \\
\hline Engenharias & 56 & 58 & 41 & 68 & 82 & 143 & 128 & 166 & 181 & 171 & 163 \\
\hline $\begin{array}{l}\text { Ciências da } \\
\text { Saúde }\end{array}$ & 49 & 59 & 56 & 65 & 49 & 112 & 174 & 246 & 211 & 237 & 147 \\
\hline $\begin{array}{l}\text { Ciências } \\
\text { Agrárias }\end{array}$ & 81 & 90 & 92 & 179 & 222 & 286 & 328 & 408 & 334 & 432 & 454 \\
\hline $\begin{array}{l}\text { Ciências Sociais } \\
\text { e Aplicadas }\end{array}$ & 37 & 42 & 48 & 43 & 65 & 102 & 158 & 166 & 186 & 163 & 127 \\
\hline $\begin{array}{l}\text { Ciências } \\
\text { Humanas }\end{array}$ & 94 & 120 & 115 & 120 & 124 & 190 & 202 & 237 & 271 & 228 & 270 \\
\hline $\begin{array}{l}\text { Linguística, } \\
\text { Letras e Arte }\end{array}$ & 07 & 14 & 17 & 27 & 43 & 60 & 49 & 70 & 84 & 64 & 59 \\
\hline Outros & 00 & 00 & 00 & 01 & 00 & 00 & 09 & 03 & 00 & 00 & 00 \\
\hline Total & 566 & 609 & 580 & 774 & 929 & 1298 & 1605 & 1955 & 1760 & 1880 & 1802 \\
\hline
\end{tabular}

Fonte: Anais EAIC - elaborada pelo autor.

Em seguida, as outras áreas que ocupam destaque são Ciências Exatas e da Terra, depois Ciências Biológicas, Ciências da Saúde e Engenharias. Essa hierarquia é semelhante à realidade tanto da UFPR como da Unicamp e, provavelmente, do conjunto das universidades públicas brasileiras.

Há destaque para a área da educação na Tabela 6 , mostrando o volume das comunicações apresentadas para as Ciências Humanas $(\mathrm{CH})$ como um todo e os trabalhos apresentados na área educacional.

Tabela 6 - Trabalhos apresentados nos EAIC na área de Educação em relação às demais áreas das Ciências Humanas $(\mathrm{CH})$

\begin{tabular}{llllllllllll}
\hline \multirow{2}{*}{ Grande Àrea $\mathrm{CH}$} & \multicolumn{10}{c}{ Ano } \\
\cline { 2 - 12 } & 1995 & 1996 & 1997 & 1998 & 1999 & 2000 & 2001 & 2002 & 2003 & 2004 & 2005 \\
\hline Educação & 20 & 20 & 21 & 24 & 35 & 61 & 30 & 29 & 71 & 66 & 47 \\
Outras Áreas (CH) & 74 & 100 & 94 & 96 & 89 & 129 & 172 & 208 & 200 & 162 & 223 \\
Total & 94 & 120 & 115 & 120 & 124 & 190 & 202 & 237 & 271 & 228 & 270 \\
\hline
\end{tabular}

Fonte: Anais EAIC - elaborada pelo autor 
Como já observamos, a IC, uma ação educacional que envolve um alto grau de envolvimento didático-científico entre professor-orientador e alunoorientando e que congrega várias vantagens para todos os setores envolvidos na sua realização, parece não mobilizar os pedagogos, a explicação de tal fenômeno merece estudos mais aprofundados, mas é possível conjecturar que tal situação é reflexo da institucionalização tardia das Faculdades de Educação no Brasil, ocorridas principalmente a partir da década de 70 (TRINDADE, 2004).

\section{Considerações finais}

Os esforços demandados pelos órgãos governamentais (CNPq e Fundações Estaduais de Amparo à Pesquisa), bem como as IES para institucionalizar e consolidar a Iniciação Científica, é um fato marcante no ensino superior que paulatinamente caminha para uma mudança qualitativa na compreensão de superar a velha concepção de reduzir a educação apenas ao seu caráter de mera transmissão passiva de conhecimentos.

Ao propor ações que incentivem um número cada vez maior de estudantes vivenciar a sua formação acadêmica para além da sala de aula, incrementando esses anos de estudos com a prática da pesquisa,éum importante passo para desdobramentos de médio e longo prazos para a sociedade em geral e os jovens em particular. Embora nossa distância em relação aos países centrais ainda é bastante desproporcional em vários aspectos, o país vem paulatinamente participando cada vez mais com publicações científicas internacionais em vários setores e é hoje o principal centro de produção científica e tecnológica da América Latina, assim a Iniciação Científica é uma importante política que reflete positivamente ao longo de todo o processo de formação da população estudantil das nossas instituições de ensino superior.

Acreditamos que o mito de que a pesquisa é privilégio de umas poucas instituições universitárias também está sendo superado. Como pudemos verificar, quando há forte empenho dos principais atores envolvidos na questão, as políticas e os programas se concretizam e mostram resultados significativos.

Nossos dados permitem afirmar que, mantidas as condições atuais, o Estado do Paraná e suas instituições estaduais de ensino superior encaminham-se para brevemente atingir níveis de excelência, registrados por universidades tradicionais como Unicamp, USP, UFPR, entre outras, no gerenciamento da Iniciação Científica, o que implica o incremento e 
diversificação maiores das formas de financiamento, no aumento quantitativo de alunos participantes em projetos de pesquisas que resultaria em continuidade de tais estudos no nível da pós-graduação.

\section{Referências}

AMARAL, N. C. Evolução do custo do aluno das IFES: eficiência? Avaliação, v. 9, n. 1, p. 115-125, 2004.

BAUMANN, Z. Desafios educacionais da modernidade líquida. Revista Tempo Brasileiro, n. 148, p. 41-58, 2002.

BREGLIA, V. L. A. A formação na graduação: contribuições, impactos e repercussões do Programa Institucional de Bolsas de Iniciação Científica (PIBIC). 2001. 159 f. Tese (Doutorado em Educação) - Pontifícia Universidade Católica do Rio de Janeiro, Rio de Janeiro, 2001.

BRIDI, J. C. A. A Iniciação Científica na formação do universitário. 2004. 135 f. Dissertação (Mestrado em Educação) - Universidade Estadual de Campinas, Campinas, 2004.

CARDOSO, G. P. Programa de Iniciação Científica. Cadernos da ABEM, v. 1, n. 1, p. 17-18, 2004.

CARVALHO, A. R.; ANGELINI, R. Variáveis determinantes do desejo de cursar mestrado de alunos de biologia (UEG, UFG e UNITINS). Avaliação, v. 7, n. 4, p. 193-202, 1996.

CASTRO, A. A. Iniciação Científica: como continuar. In: GOLDENBERG, S.; GUimarÃES, C. A.; CASTRO, A. A. Elaboração e Apresentação de Comunicação Científica. São Paulo: Metodologia, 2002-2007. Disponível em: $<$ http://www.metodologia.org >. Acesso em: 25 set. 2007.

CATANI, A. M.; OLIVEIRA, J. F. A reestruturação da educação superior no debate internacional: a padronização das políticas de diversificação e diferenciação. Revista Portuguesa de Educação, v. 13, n. 2, p. 29-52, 2000.

CONGRESSO INTERNO DE INICIAÇÃO CIENTÍFICA. 14., 2006, Campinas. Anais... Campinas: Universidade Estadual de Campinas, 2006. 
ENCONTRO ANUAL DE INICIAÇÃO CIENTÍFICA. 7., 1998, Maringá. Anais... Maringá: Universidade Estadual de Maringá, 1998.

ENCONTRO ANUAL DE INICIAÇÃO CIENTÍFICA. 8., 1999, Cascavel. Anais... Cascavel: Universidade Estadual de Oeste do Paraná, 1999.

ENCONTRO ANUAL DE INICIAÇÃO CIENTÍFICA. 9., 2000, Londrina. Anais... Londrina: Universidade Estadual de Londrina, 2000.

ENCONTRO ANUAL DE INICIAÇÃO CIENTÍFICA. 15., 2006, Ponta Grossa. Anais... Ponta Grossa: Universidade Estadual de Ponta Grossa, 2006.

EVENTO DE INICIAÇÃO CIENTÍFICA. 13., 2005, Curitiba. Anais... Curitiba: Universidade Federal do Paraná, 2005.

FAVA-DE-MORAES, F.; FAVA, M. A Iniciação Científica: muitas vantagens e poucos riscos. São Paulo em Perspectiva, v. 14, n. 1, 73-77, 2000.

GIANOTTI, J. A. Capitalismo e monopólio do conhecimento. Novos Estudos CEBRAP, n. 64, 107-120, 2002.

INSTITUTO NACIONAL DE ESTUDOS E PESQUISAS EDUCACIONAIS ANÍSIO TEIXEIRA (INEP). Censo da educação superior 2006. Disponível em: <http://www.inep.gov.br>. Acesso em: 16 out. 2007.

LOCH, J. M.; REIS, D. R. A expansão do ensino superior: um comparativo do crescimento no Brasil, no Paraná e na região metropolitana de Curitiba. In: World Congress on Engineering and Technology Education. 1., 2004, Santos. Anais... Santos: UniSantos, 2004. p. 1605-1608.

MALDONADO, L. A.; PAIVA, E. V. A Iniciação Científica na graduação em Nutrição: possibilidades e contribuições para a formação profissional. In: CALAZANS, M. J. C. Iniciação Científica: construindo o pensamento crítico. São Paulo: Cortez, 2002. p. 141-162.

ORGANISATION FOR ECONOMIC CO-OPERATION AND DEVELOPMENT (OECD). Relatório de auto-análise da região Norte do Paraná. Disponível em: <http://www.oecd.org>. Acesso em: 22 nov. 2007.

PRÓ-REITORIA DE PLANEJAMENTO-PROPLAN. Plano de Desenvolvimento Institucional - PDI. Disponível em: <http://www.uel.br/ proplan;http://www.uem.br>. Acesso em: 15 abr. 2007. 
SAMPAIO, H. Ensino superior no Brasil: o setor privado. São Paulo: HUCITEC, 2000.

SECRETARIA DE ESTADO DA CIÊNCIA, TECNOLOGIA E ENSINO SUPERIOR (SETI). Mapa das IEES. Disponível em: < http://www.seti.pr.gov. br>. Acesso em: 28 abr. 2007.

SANTOS, C. R. A. Apresentação. In: APPEL, E. A Universidade na encruzilhada Seminário Universidade: por que e como reformar? Brasilia: Unesco, 2003. p. 9-20.

SETTON, M. G. J. Diferenças e semelhanças entre os alunos de Humanidades da USP: vida acadêmica e profissional. Avaliação, v. 8, n. 3, p. 93-132, 2003.

SILVA, R. de C.; CABRERO, R. de C. Iniciação Científica: rumo à pós-graduação. Educação Brasileira, v. 20, n. 40, 189-199, 1998.

SOUZA, S. M. Z.; OLIVEIRA, R. P.; GONÇALVES, N. G. Evasão dos alunos do Programa de pós-graduação da FEUSP: 1990-2000. Avaliação, v. 8, n. 3, p. 191-228, 2001.

TRINDADE, H. H. C. A República em tempos de reforma universitária: os desafios do Governo Lula. Educação \& Sociedade, v. 25, n. 88, p. 819-844, 2004.

VELLOSO, J. R.; VELHO, L. M. L. S. Mestrandos e doutorandos no país: trajetórias de formação. Brasília: CAPES, 2001.

VIANNA, H. M. Avaliação de cursos pelos alunos: considerações. Estudos em Avaliação Educacional, n. 29, p. 137-148, 2004.

VILLAS BÔAS, G. K. Currículo, iniciação científica e evasão de estudantes de Ciências Sociais. Tempo Social, v. 15, n. 1, v. 15, p. 45-62, 2003.

YAMAMOTO, M. E.; FERNANDES JUNIOR, V. J. Bases de pesquisa: a experiência da UFRN no fomento institucional de pesquisa. In: CALAZANS, M. J. C. Iniciação Científica: construindo o pensamento crítico. São Paulo: Cortez, 2002. p. 161-180.

Recebido: 10/04/2009

Received: 04/10/2009

Aprovado: 12/06/2009

Approved: 06/12/2009 